OPEN ACCESS

Edited by:

Haigang Ren,

Soochow University, China

Reviewed by:

Bijay Parajuli,

University of Yamanashi, Japan

*Correspondence:

Guy C. Brown

gcb3@cam.ac.uk

Specialty section:

This article was submitted to Alzheimer's Disease and Related

Dementias,

a section of the journa

Frontiers in Aging Neuroscience

Received: 13 December 2021 Accepted: 31 December 2021

Published: 28 January 2022

Citation:

Brown GC and St George-Hyslop P (2022) Does Soluble TREM2 Protect Against Alzheimer's Disease?

Front. Aging Neurosci. 13:834697.

doi: 10.3389/fnagi.2021.834697

\section{Does Soluble TREM2 Protect Against Alzheimer's Disease?}

\author{
Guy C. Brown ${ }^{1 *}$ and Peter St George-Hyslop ${ }^{2,3}$ \\ ${ }^{1}$ Department of Biochemistry, University of Cambridge, Cambridge, United Kingdom, ${ }^{2}$ Department of Medicine, University of \\ Cambridge, Cambridge, United Kingdom, ${ }^{3}$ Department of Medicine, University of Toronto, Toronto, ON, Canada
}

Triggering Receptor Expressed in Myeloid Cells 2 (TREM2) is a pattern recognition receptor on myeloid cells, and is upregulated on microglia surrounding amyloid plaques in Alzheimer's disease (AD). Rare, heterozygous mutations in TREM2 (e.g., R47H) increase $A D$ risk several fold. TREM2 can be cleaved at the plasma membrane by metalloproteases to release the ectodomain as soluble TREM2 (sTREM2). Wild-type sTREM2 binds oligomeric amyloid beta $(A \beta)$ and acts as an extracellular chaperone, blocking and reversing $A \beta$ oligomerization and fibrillization, and preventing $A \beta$-induced neuronal loss in vitro. Whereas, R47H sTREM2 increases $A \beta$ fibrillization and neurotoxicity. AD brains expressing R47H TREM2 have more fibrous plaques with more neuritic pathology around these plaques, consistent with R47H sTREM2 promoting A $\beta$ fibrillization relative to WT sTREM2. Brain expression or injection of wild-type sTREM2 reduces pathology in amyloid models of $A D$ in mice, indicating that wild-type sTREM2 is protective against amyloid pathology. Levels of sTREM2 in cerebrospinal fluid (CSF) fall prior to $A D$, rise in early $A D$, and fall again in late $A D$. People with higher sTREM2 levels in CSF progress more slowly into and through $A D$ than do people with lower sTREM2 levels, suggesting that sTREM2 protects against AD. However, some of these experiments can be interpreted as full-length TREM2 protecting rather than sTREM2, and to distinguish between these two possibilities, we need more experiments testing whether sTREM2 itself protects in AD and AD models, and at what stage of disease. If sTREM2 is protective, then treatments could be designed to elevate sTREM2 in AD.

Keywords: TREM2, sTREM2, microglia, Alzheimer's disease, amyloid beta, neuroinflammation, neurodegeneration, neuroprotection

\section{INTRODUCTION}

\section{TREM2}

Triggering Receptor Expressed in Myeloid Cells 2 (TREM2) is a pattern recognition receptor found on the plasma membrane of myeloid cells. When activated by ligands, such as phospholipids, lipoproteins, and amyloid beta peptide (A $\beta)$, TREM2 induces an innate immune response, which includes phagocytosis, chemotaxis, and transcriptional changes (Keren-Shaul et al., 2017; Deczkowska et al., 2020; Kulkarni et al., 2021). TREM2 signaling is mainly via binding DAP12 (DNAX-activating protein of $12 \mathrm{kDa}$ ), which activates Syk tyrosine kinase (Deczkowska et al., 2020). Within the brain, TREM2 is almost uniquely expressed by microglia, and is upregulated on microglia around amyloid plaques in AD (Giraldo et al., 2013; Yuan et al., 2016; Brendel et al., 2017). Rare, heterozygous mutations of TREM 2 are known to affect AD risk, including the R47H 
mutation, which increases $\mathrm{AD}$ risk several fold (Guerreiro et al., 2012; Giraldo et al., 2013; Jonsson et al., 2013; Kulkarni et al., 2021). These mutations are thought to increase AD risk by reducing the protective roles of microglial TREM2, in particular by reducing microglial phagocytosis of amyloid plaques (Condello et al., 2015; Yuan et al., 2016).

\section{STREM2}

TREM2 is a single-pass type I transmembrane protein with a small C-terminal on the cytosolic side of the plasma membrane, and an N-terminal ectodomain that includes the ligand binding site (Zhong and Chen, 2019; Yang et al., 2020). However, the ectodomain of TREM2 is shed from cells expressing full-length TREM2 into the extracellular medium, and is then known as soluble TREM2 (sTREM2) (Piccio et al., 2008; Wunderlich et al., 2013). The turnover of full-length TREM2 on macrophages is very rapid with a half-life of $<1 \mathrm{~h}$, because of constitutive cleavage of full-length TREM2 and shedding of sTREM2 (Thornton et al., 2017). The proteases responsible for shedding sTREM2 include A Disintegrin And Metalloproteases 10 and 17 (ADAM10 and ADAM17), and this cleavage occurs at the H157-S158 peptide bond (Schlepckow et al., 2017; Thornton et al., 2017). ADAM10 and 17 appear to be responsible for sTREM2 release induced by lipopolysaccharide (LPS), whereas the protease meprin $\beta$ constitutively cleaves TREM2 (predominately at the R136-D137 peptide bond) to release sTREM2 from macrophages (Berner et al., 2020). However, it is unclear whether meprin $\beta$ can generate sTREM2 in microglia. After shedding of sTREM2, the remaining part of TREM2 may be cleaved within the membrane by $\gamma$ secretase (Wunderlich et al., 2013). The very rapid and inducible turnover of TREM2 to generate sTREM2 suggests either that (i) TREM2 levels need to be regulated very rapidly, or (ii) that sTREM2 has a function, and full-length TREM2 is a precursor of this functional sTREM2.

\section{Regulation of sTREM2 Shedding}

Conditions that increase or decrease sTREM2 shedding from full-length TREM2 are not clear, but LPS or IL- $1 \beta$ can induce sTREM2 release from primary mouse microglia (Zhong et al., 2019). Also, oligomeric $A \beta$, which can bind both full-length TREM2 and sTREM2, induced shedding of sTREM2 for TREM2overexpressing cells (Vilalta et al., 2021), suggesting that sTREM2 shedding may be induced prior to and during $\mathrm{AD}$ as a result of $\mathrm{A} \beta$ oligomerization. CSF sTREM2 levels increase in amyloid mouse models and correlate with microglial activation (Brendel et al., 2017). Viral infection of the lungs can increase sTREM2 levels post-infection, due to IL-13 or IL-4 induced sTREM2 shedding (Wu et al., 2015). And HIV viral infection of the brain increases CSF levels of sTREM2 (Gisslén et al., 2018). sTREM2 levels in CSF are thought to be a biomarker of microglial activation, although there is limited evidence for this in vivo (Bekris et al., 2018; Rauchmann et al., 2020; Pascoal et al., 2021), and sTREM2 may itself cause microglial activation (see below). CSF sTREM2 levels rise with age in humans from about $2 \mathrm{ng} / \mathrm{ml}$ at 43 years to $6 \mathrm{ng} / \mathrm{ml}$ at 80 years of age (Henjum et al., 2016).

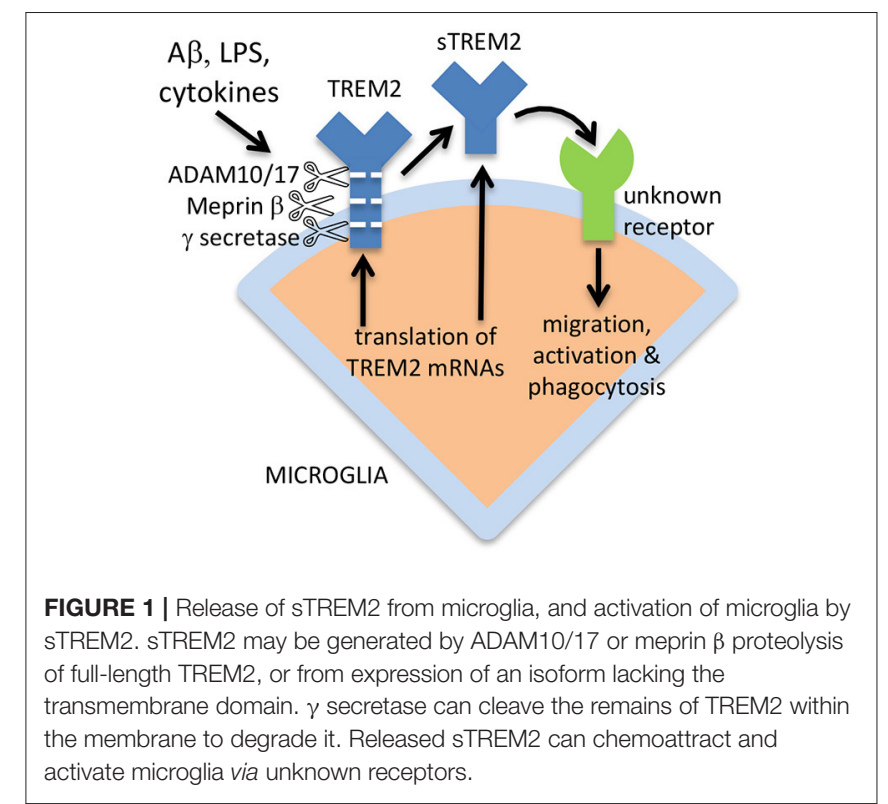

\section{Alternative Forms of sTREM2}

TREM2 can be expressed via alternative splicing as a soluble isoform, lacking the transmembrane form, and this alternative sTREM 2 may constitute $25 \%$ of total sTREM2 in the brain (Ma et al., 2016; Del-Aguila et al., 2019). This again suggests that sTREM2 has a function, rather than being simply a degradation product of full-length TREM2. The sTREM2 generated by alternative splicing would be 219 amino acids residues long, the sTREM2 generated by ADAM10 or 17 would be 157 amino acids residues long, and the sTREM2 generated by meprin $\beta$ would be 136 amino acids residues long (plus shorter forms) (Berner et al., 2020), although removal of the signal peptide would shorten all these sTREM2 forms by 18 amino acid residues. The ectodomain of TREM2 and sTREM2 is highly glycosylated at Asn20 and Asn79, so the apparent molecular weight of fulllength TREM 2 on electrophoresis gels is about $50 \mathrm{kDa}$ when fully glycosylated, and about $25 \mathrm{kDa}$ when deglycosylated (Ma et al., 2016). The apparent molecular weight of sTREM2 in CSF is 30$35 \mathrm{kDa}$ (Ma et al., 2016), implying that almost half the apparent weight of sTREM2 is sugars, and that different glycosylation states coexist. The alternative mechanisms of sTREM2 generation are illustrated in Figure 1.

\section{sTREM2 Degradation}

Processes responsible for degradation and clearance of extracellular sTREM2 are unclear, although it has been found that macrophages readily take up sTREM2 (Wu et al., 2015), and sTREM2 injected into mouse brain is cleared from the brain within 3 days (Zhong et al., 2019). Membrane-attached meprin $\beta$ generates sTREM2 constitutively, but inflammationinduced ADAM10/17 releases soluble meprin $\beta$, which can rapidly degrade sTREM2 (Berner et al., 2020). However, it is unclear whether meprin $\beta$ contributes to sTREM2 production or degradation in the brain. 

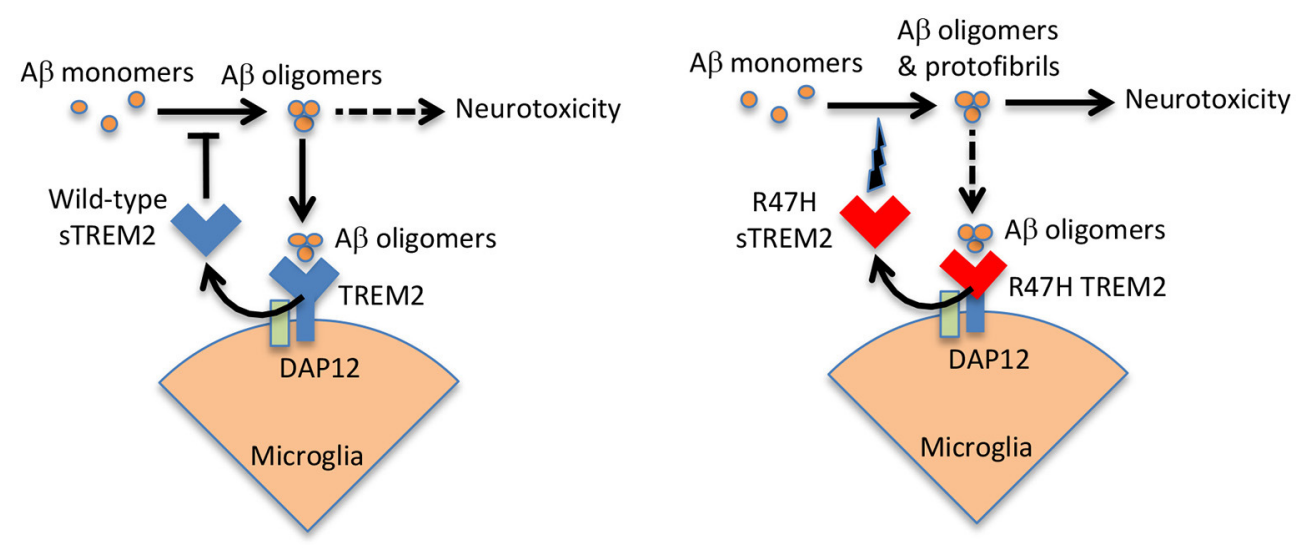

FIGURE 2 | Wild-type sTREM2 blocks A $\beta$ pathology, but R47H TREM2 does the opposite. A $\beta$ oligomers bind to TREM2 and induce shedding of sTREM2. Wild-type sTREM2 blocks A $\beta$ oligomerization, fibrillization and neurotoxicity. R47H sTREM2 increases A $\beta$ oligomerization, fibrillization and neurotoxicity. Thus, wild-type sTREM2 may protect against amyloid pathology, while R47H TREM2 exacerbates amyloid pathology. This might help explain why a single copy of the R47H TREM2 gene increases $A D$ risk several fold.

\section{ACTIONS OF STREM2}

\section{sTREM2 Activates Microglia}

sTREM2 treatment of macrophages induced phosphorylation of ERK1/2 (extracellular signal-regulated kinases 1 and 2) and inhibited apoptosis (Wu et al., 2015). Similarly, sTREM2 treatment of microglia in culture promoted survival by inhibiting apoptosis, apparently via activation of Akt (Zhong et al., 2017). In addition, sTREM2 induced inflammatory activation of cultured microglia via nuclear factor- $\kappa \mathrm{B}$, resulting in morphological activation and release of pro-inflammatory cytokines (Zhong et al., 2017). sTREM2 also stimulated migration and phagocytosis by primary microglia in culture (Zhong et al., 2019). Injection of sTREM2 into the brains of mice expressing the amyloid precursor protein (APP) induced activation and proliferation of microglia, plus increased expression of pro-inflammatory cytokines, and increased microglial phagocytosis of $\mathrm{A} \beta$ (Zhong et al., 2019). Injection of sTREM2 into the brains of healthy mice also induced expression of pro-inflammatory cytokines (Fassler et al., 2021). A fragment of sTREM2 (amino acids 51-81) was sufficient to activate microglia (Sheng et al., 2021). Thus, sTREM2 activates microglia, although the mechanism of this activation is unclear.

\section{sTREM2 Blocks A $\beta$ Aggregation and Neurotoxicity}

sTREM2 is known to bind oligomeric $A \beta$, with minimal binding to monomeric or fibrillar $\mathrm{A} \beta$ (Lessard et al., 2018; Zhao et al., 2018; Zhong et al., 2018; Vilalta et al., 2021). Subsequently, it was found that sTREM2 blocked $A \beta$ oligomerisation and fibrillization at a molar ratio of 1 sTREM2 to $100 \mathrm{~A} \beta$ (Kober et al., 2021; Vilalta et al., 2021), and at higher molar ratios sTREM2 disaggregated $A \beta$ oligomers and fibrils (Vilalta et al., 2021). Wild-type sTREM2 also inhibited $A \beta$-induced permeabilization of artificial membranes, and inhibited $A \beta$-induced neuronal loss in glial-neuronal cultures (Vilalta et al., 2021). These results suggest that wild-type sTREM2 may act as extracellular chaperone for $A \beta$, blocking its folding into aggregatable forms and refolding aggregates into soluble forms, thereby inhibiting the neurotoxicity of $A \beta$. In contrast, R47H sTREM2 bound less to $A \beta$ oligomers, but increased $A \beta$ aggregation into protofibrils, and increased $A \beta$-induced neuronal loss in glial-neuronal cultures (Vilalta et al., 2021). Thus, R47H sTREM2 may not only loose a neuroprotective function, but also gain a neurotoxic function in the presence of $A \beta$, probably by folding $A \beta$ into more toxic forms (see Figure 2).

\section{sTREM2 Protects Against Amyloid Pathology in Mice}

sTREM2 injection into the brains of mice expressing APP reduced amyloid plaque load (Zhong et al., 2019). Furthermore, viral expression of sTREM2 in the APP-expressing mice, reduced plaque load and reversed deficits of spatial memory and longterm potentiation (Zhong et al., 2019). Thus, sTREM2 is protective against amyloid pathology in mice, and this might be by sTREM2 affecting $A \beta$ aggregation and/or sTREM2 activating microglia to phagocytose plaques. A fragment of sTREM2 (amino acids 51-81) was sufficient to activate microglia, but not to bind $\mathrm{A} \beta$ and reduce amyloid pathology in vivo; whereas a 41-81 fragment of sTREM 2 bound $A \beta$ and reduced amyloid pathology in vivo better than full-length sTREM2 (Sheng et al., 2021). This suggests that sTREM2 protects against amyloid pathology mainly by binding $A \beta$.

TREM2 knockout mice, crossed with APP-expressing mice, have more fibrous and less compact plaques (Condello et al., 2015; Wang et al., 2016; Yuan et al., 2016; Song et al., 2018), and while this has been attributed to less microglial phagocytosis of the plaques because of less full-length TREM2, the result might alternatively be due to sTREM2 blocking $\mathrm{A} \beta$ aggregation and/or sTREM2 activating microglia to phagocytose plaques. TREM2 knockout mice have increased $A \beta$ seeding (Parhizkar et al., 2019), which again could be explained by reduced microglial phagocytosis of $A \beta$ seeds mediated by full-length TREM2, or 
reduced blocking of $A \beta$ aggregation by sTREM2. In 5xFAD mice expressing wild-type human TREM2, sTREM2 was found bound to the amyloid plaques (Song et al., 2018), consistent with sTREM2 having a role in regulating plaques. Note that the ability of sTREM2 to block $A \beta$ aggregation and to disaggregate $\mathrm{A} \beta$, might be shared with full-length TREM2, as they both bind $\mathrm{A} \beta$ oligomers (Vilalta et al., 2021), but this has not been tested. Humans (and mice) with heterozygous R47H TREM2 have more fibrous plaques with more neuritic pathology (Yuan et al., 2016), which again might be explained by either $\mathrm{R} 47 \mathrm{H}$ sTREM2 promoting A $\beta$ fibrillation, or by reduced microglial phagocytosis of plaques.

\section{EVIDENCE THAT STREM2 IS PROTECTIVE AGAINST AD IN HUMANS}

CSF levels of sTREM2 fall significantly in early pre-symptomatic stages prior to $\mathrm{AD}$ diagnosis (when amyloid is aggregating), but rise during mild cognitive impairment $(\mathrm{MCI})$ and $\mathrm{AD}$ (when tau is aggregating), and fall again during the dementia stages of $\mathrm{AD}$ (Heslegrave et al., 2016; Piccio et al., 2016; Suárez-Calvet et al., 2016, 2019; Bekris et al., 2018; Liu et al., 2018; Nordengen et al., 2019; Rauchmann et al., 2019; Ma et al., 2020). People with higher CSF levels of sTREM2 progress more slowly through MCI and $\mathrm{AD}$, in terms of memory loss, clinical score and brain atrophy (Ewers et al., 2019, 2020; Edwin et al., 2020; Franzmeier et al., 2020). And this apparent protective effect of sTREM2 correlated with reduced amyloid and Tau aggregation measured by PET (Ewers et al., 2020), consistent with sTREM2 reducing amyloid aggregation and pathology.

However, these apparent protective effect of high sTREM2 has been attributed to full-length TREM2, rather than STREM2, on the untested assumption that high sTREM2 levels indicates high TREM2 levels, as a result of constant shedding. However, if elevated sTREM2 results from elevated shedding, which is for example induced by oligomeric A $\beta$ (Vilalta et al., 2021), then this will reduce full-length TREM2. Thus, elevated levels of sTREM2 do not necessarily indicate that levels of full-length TREM2 are elevated, and the apparent protective effect of sTREM2 against $\mathrm{AD}$ may be more simply explained by sTREM2 itself being protective.

GWAS studies of gene variants that affect the CSF levels of sTREM2 identified the membrane-spanning 4-domains superfamily A (MS4A) gene cluster as key determinants of sTREM2 levels in CSF (Piccio et al., 2016; Deming et al., 2019; Hou et al., 2019). This gene region had previously been linked to $\mathrm{AD}$ risk (Naj et al., 2011). For example, rs1582763 increased brain expression of MS4A4A and MS4A6A genes, increased sTREM2 levels in CSF, reduced $\mathrm{AD}$ risk and increased age of $\mathrm{AD}$ diagnosis. While rs6591561 resulted in a loss-of-function $M S 4 A 4 A$, reduced CSF sTREM2 levels, increased AD risk and reduced age at $\mathrm{AD}$ onset (Deming et al., 2019). MS4A4A and TREM2 were found to colocalize at the plasma membrane, and overexpression of MS4A4A increased sTREM2 levels, whilst silencing of MS4A4A reduced sTREM2 levels (Deming et al., 2019). This suggests that MS4A4A may affect AD risk by promoting sTREM2 shedding, and if so, indicating that sTREM2, rather than full-length TREM2 is protective against $\mathrm{AD}$. However, further work is required to establish whether MS4A4A directly affects sTREM2 shedding.

\section{EVIDENCE AGAINST THE HYPOTHESIS THAT STREM2 PROTECTS}

One piece of evidence potentially contradicting a protective role of sTREM2 in AD, is that the H157Y mutation of TREM2 expressed in cells significantly increased sTREM2 shedding relative to wild-type TREM2, resulting in increased sTREM2 and decreased full-length TREM2, but is associated with increased AD risk (Schlepckow et al., 2017; Thornton et al., 2017). This suggests that the increased AD risk associated with the H157Y mutation is due to decreased full-length TREM2 or increased sTREM2, contradicting the hypothesis that sTREM2 is protective against $\mathrm{AD}$. However, the $\mathrm{H} 157 \mathrm{Y}$ mutation only increased shedding by about 50\%, and this was from HEK293 cells (Schlepckow et al., 2017; Thornton et al., 2017), so it may be difficult to extrapolate to sTREM2 levels in human brains. Additionally, the H157Y mutation would constitute the C-terminal of sTREM2, and might affect its properties, such as its interactions with $A \beta$. Thus, it would be important to determine whether this mutation does indeed increase CSF levels of sTREM2 in humans, and whether H157Y sTREM2 has the same protective properties as wild-type sTREM2.

Other evidence potentially contradicting the hypothesis that sTREM2 protects against AD is the finding of Schlepckow et al. (2020) that an antibody binding to the ADAM cleavage site of TREM2 prevented sTREM2 release, but reduced plaques load in an amyloid mouse model. However, the antibody used directly activated TREM2 signaling, so the reduced plaque load may result from this signaling (Schlepckow et al., 2020). Additionally, the compaction of these plaques, neuritic pathology and memory loss were not tested in this model.

\section{DISCUSSION}

\section{Is TREM2 or STREM2 Protective in Alzheimer's Disease?}

It appears that either TREM2 or sTREM2 are protective in Alzheimer's disease, but which? TREM2 is thought to be protective by (i) recruiting and activating microglia into a protective state around amyloid plaques, and (ii) compacting amyloid plaques by phagocytosis of $\mathrm{A} \beta$, preventing the plaques inducing neuritic pathology (Condello et al., 2015; Yuan et al., 2016; Keren-Shaul et al., 2017). Whereas, sTREM2 is thought to be protective by: (i) stimulating microglial recruitment, activation and phagocytosis of $\mathrm{A} \beta$, and/or (ii) blocking and reversing $A \beta$ aggregation, preventing neurotoxicity (Zhong et al., 2019; Vilalta et al., 2021). Thus, the putative protective effects of TREM2 and sTREM2 are complimentary rather than antagonistic, and potentially both may be protective against Alzheimer's disease. However, it is still important to verify that TREM2 and/or sTREM2 are in fact protective. 


\section{Key Experiments to Determine Whether sTREM2 Is Protective Against AD}

Some of evidence indicating that sTREM2 is protective against $\mathrm{AD}$, may alternatively be interpreted as full-length TREM2 is protective. Thus, there is a need for experiments that distinguish between these possibilities, or directly show that sTREM2 is protective. The most direct way to show that is to add or express sTREM2 independent of full-length TREM2 and test whether this is protective in AD models. This has been done for a mouse amyloid model and found to be protective (Zhong et al., 2019), but this was relatively acute model, and it would be important to test this in other models, particularly more chronic and AD-relevant models. Within such models, it would be important to test whether sTREM 2 can block $A \beta$ aggregation, or disaggregate preformed plaques or oligomers. It would also be useful to know whether A $\beta$ oligomers in AD CSF are significantly bound to sTREM2, and whether physiological levels of sTREM2 can disaggregate $A \beta$ aggregation in CSF. Further, it would be worth knowing whether the different types of sTREM2 behave differently, including sTREM2 generated by ADAM and meprin $\beta$, or by alternative splicing, or H157Y and R62H sTREM2.

\section{Potential Treatment Strategies}

Current strategies targeting TREM2 in AD have focused on agonistic antibodies to activate TREM2 with the aim of increasing microglial phagocytosis of amyloid plaques (Wang et al., 2020; Fassler et al., 2021). These antibodies will also bind sTREM2 and potentially block the protective effects of sTREM2 (Fassler et al., 2021). If sTREM2 is indeed more protective against

\section{REFERENCES}

Bekris, L. M., Khrestian, M., Dyne, E., Shao, Y., Pillai, J. A., Rao, S. M., et al. (2018). Soluble TREM2 and biomarkers of central and peripheral inflammation in neurodegenerative disease. J. Neuroimmunol. 319, 19-27. doi: 10.1016/j.jneuroim.2018.03.003

Berner, D. K., Wessolowski, L., Armbrust, F., Schneppenheim, J., Schlepckow, K., Koudelka, T., et al. (2020). Meprin $\beta$ cleaves TREM2 and controls its phagocytic activity on macrophages. FASEB J. 34, 6675-6687. doi: 10.1096/fj.2019 02183R

Brendel, M., Kleinberger, G., Probst, F., Jaworska, A., Overhoff, F., Blume, T., et al. (2017). Increase of TREM2 during aging of an Alzheimer's disease mouse model is paralleled by microglial activation and amyloidosis. Front. Aging Neurosci. 9:8. doi: 10.3389/fnagi.2017.00008

Condello, C., Yuan, P., Schain, A., and Grutzendler, J. (2015). Microglia constitute a barrier that prevents neurotoxic protofibrillar A $\beta 42$ hotspots around plaques. Nat. Commun. 6:6176. doi: 10.1038/ ncomms7176

Deczkowska, A., Weiner, A., and Amit, I. (2020). The physiology, pathology, and potential therapeutic applications of the TREM2 signaling pathway. Cell 181, 1207-1217. doi: 10.1016/j.cell.2020.05.003

Del-Aguila, J. L., Benitez, B. A., Li, Z., Dube, U., Mihindukulasuriya, K. A., Budde, J. P., et al. (2019). TREM2 brain transcript-specific studies in AD and TREM2 mutation carriers. Mol. Neurodegener. 14:18. doi: 10.1186/s13024-019-0 319-3

Deming, Y., Filipello, F., Cignarella, F., Cantoni, C., Hsu, S., Mikesell, R., et al. (2019). The MS4A gene cluster is a key modulator of soluble
AD than full-length TREM2, then antibodies that increased sTREM2 shedding might be beneficial, or other treatments designed to activate sTREM2 shedding e.g., by activating ADAM10 and ADAM17. Blocking sTREM2 degradation (e.g., by inhibiting meprin $\beta$ ) might increase sTREM2 levels without decreasing full-length TREM2. sTREM2 and sTREM2 fragments injected into the brain were protective in mouse models of $\mathrm{AD}$ (Zhong et al., 2019; Sheng et al., 2021), but may be difficult to deliver practically in humans. However, viral vectors expressing sTREM2 in the brain were protective in these mouse models of $\mathrm{AD}$, and thus might be protective in humans with $\mathrm{AD}$ (Zhong et al., 2019).

\section{AUTHOR CONTRIBUTIONS}

GB wrote the article. PG-H reviewed and adjusted the article. Both authors were responsible for its content.

\section{FUNDING}

This project has received funding from the Innovative Medicines Initiative 2 Joint Undertaking under Grant Agreement No. 115976. This Joint Undertaking receives support from the European Union's Horizon 2020 Research and Innovation Programme and EFPIA. PHStGH also received funding from the Canadian Institutes of Health Research (Foundation Grant and Canadian Consortium on Neurodegeneration in Aging Grant), Wellcome Trust Collaborative Award 203249/Z/16/Z, US Alzheimer's Society Zenith Grant ZEN-18-529769, and the Alzheimer's Society of Ontario Chair in Alzheimer's Disease Research.
TREM2 and Alzheimer's disease risk. Sci. Transl. Med. 11:eaau2291. doi: 10.1126/scitranslmed.aau2291

Edwin, T. H., Henjum, K., Nilsson, L. N. G., Watne, L. O., Persson, K., Eldholm, R. S., et al. (2020). A high cerebrospinal fluid soluble TREM2 level is associated with slow clinical progression of Alzheimer's disease. Alzheimers Dement. 12:e12128. doi: 10.1002/dad2.12128

Ewers, M., Biechele, G., Suárez-Calvet, M., Sacher, C., Blume, T., Morenas-Rodriguez, E., et al. (2020). Higher CSF sTREM2 and microglia activation are associated with slower rates of beta-amyloid accumulation. EMBO Mol. Med. 12:e12308. doi: 10.15252/emmm.202 012308

Ewers, M., Franzmeier, N., Suárez-Calvet, M., Morenas-Rodriguez, E., Caballero, M. A. A., Kleinberger, G., et al. (2019). Increased soluble TREM2 in cerebrospinal fluid is associated with reduced cognitive and clinical decline in Alzheimer's disease. Sci. Transl. Med. 11:eaav6221. doi: 10.1126/scitranslmed.aav6221

Fassler, M., Rappaport, M. S., Cuño, C. B., and George, J. (2021). Engagement of TREM2 by a novel monoclonal antibody induces activation of microglia and improves cognitive function in Alzheimer's disease models. J. Neuroinflamm. 18:19. doi: 10.1186/s12974-020-01980-5

Franzmeier, N., Suárez-Calvet, M., Frontzkowski, L., Moore, A., Hohman, T. J., Morenas-Rodriguez, E., et al. (2020). Higher CSF sTREM2 attenuates ApoE4related risk for cognitive decline and neurodegeneration. Mol. Neurodegener. 15:57. doi: 10.1186/s13024-020-00407-2

Giraldo, M., Lopera, F., Siniard, A. L., Corneveaux, J. J., Schrauwen, I., Carvajal, J., et al. (2013). Variants in triggering receptor expressed on myeloid cells 2 are associated with both behavioral variant frontotemporal 
lobar degeneration and Alzheimer's disease. Neurobiol. Aging 34, 2077.e11-8. doi: 10.1016/j.neurobiolaging.2013.02.016

Gisslén, M., Heslegrave, A., Veleva, E., Yilmaz, A., Andersson, L. M., Hagberg, L., et al. (2018). CSF concentrations of soluble TREM2 as a marker of microglial activation in HIV-1 infection. Neurol. Neuroimmunol. Neuroinflamm. 6:e512. doi: 10.1212/NXI.00000000000 00512

Guerreiro, R., Wojtas, A., Bras, J., Carrasquillo, M., Rogaeva, E., Majounie, E., et al. (2012). TREM2 variants in Alzheimer's disease. N. Engl. J. Med. 368, 117-127. doi: 10.1056/NEJMoa1211851

Henjum, K., Almdahl, I. S., Årskog, V., Minthon L, Hansson, O., Fladby, T., and Nilsson, L. N. (2016). Cerebrospinal fluid soluble TREM2 in aging and Alzheimer's disease. Alzheimers. Res. Ther. 8:17. doi: 10.1186/s13195-016-0182-1

Heslegrave, A., Heywood, W., Paterson, R., Magdalinou, N., Svensson, J., Johansson, P., et al. (2016). Increased cerebrospinal fluid soluble TREM2 concentration in Alzheimer's disease. Mol. Neurodegener. 11:3. doi: 10.1186/s13024-016-0071-x

Hou, X. H., Bi, Y. L., Tan, M. S., Xu, W., Li, J. Q., Shen, X. N., et al. (2019). Genome-wide association study identifies Alzheimer's risk variant in MS4A6A influencing cerebrospinal fluid sTREM2 levels. Neurobiol. Aging 84, 241.e13-241.e20. doi: 10.1016/j.neurobiolaging.2019.05.008

Jonsson, T., Stefansson, H., Steinberg, S., Jonsdottir, I., Jonsson, P. V., Snaedal, J., et al. (2013). Variant of TREM2 associated with the risk of Alzheimer's disease. N. Engl. J. Med. 368, 107-116. doi: 10.1056/NEJMoa1211103

Keren-Shaul, H., Spinrad, A., Weiner, A., Matcovitch-Natan, O., Dvir-Szternfeld, R., Ulland, T. K., et al. (2017). A unique microglia type associated with restricting development of Alzheimer's disease. Cell 169, 1276-1290.e17. doi: 10.1016/j.cell.2017.05.018

Kober, D. L., Stuchell-Brereton, M. D., Kluender, C. E., Dean, H. B., Strickland, M. R., Steinberg, D. F., et al. (2021). Functional insights from biophysical study of TREM2 interactions with apoE and A $\beta 1-42$. Alzheimers Dement. 17, 475-488. doi: 10.1101/2020.02.24.963264

Kulkarni, B., Kumar, D., Cruz-Martins, N., and Sellamuthu, S. (2021). Role of TREM2 in Alzheimer's disease: a long road ahead. Mol. Neurobiol. 58, 5239-5252. doi: 10.1007/s12035-021-02477-9

Lessard, C. B., Malnik, S. L., Zhou, Y., Ladd, T. B., Cruz, P. E., Ran, Y., et al. (2018). High-affinity interactions and signal transduction between $A \beta$ oligomers and TREM2. EMBO Mol. Med. 10:e9027. doi: 10.15252/emmm.201809027

Liu, D., Cao, B., Zhao, Y., Huang, H., McIntyre, R. S., Rosenblat, J. D., et al. (2018). Soluble TREM2 changes during the clinical course of Alzheimer's disease: a meta-analysis. Neurosci. Lett. 686, 10-16. doi: 10.1016/j.neulet.2018.0 8.038

Ma, L., Allen, M., Sakae, N., Ertekin-Taner, N., Graff-Radford, N. R., Dickson, D. W., et al. (2016). Expression and processing analyses of wild type and p.R47H TREM2 variant in Alzheimer's disease brains. Mol. Neurodegener. 11:72. doi: 10.1186/s13024-016-0137-9

Ma, L. Z., Tan, L., Bi, Y. L., Shen, X. N., Xu, W., Ma, Y. H., et al. (2020). Dynamic changes of CSF sTREM2 in preclinical Alzheimer's disease: the CABLE study. Mol. Neurodegener. 15:25. doi: 10.1186/s13024-020-00374-8

Naj, A. C., Jun, G., Beecham, G. W., Wang, L. S., Vardarajan, B. N., Buros, J., et al. (2011). Common variants at MS4A4/MS4A6E, CD2AP, CD33 and EPHA1 are associated with late-onset Alzheimer's disease. Nat. Genet. 43, 436-441. doi: 10.1038/ng.801

Nordengen, K., Kirsebom, B. E., Henjum, K., Selnes, P., Gísladóttir, B., Wettergreen, M., et al. (2019). Glial activation and inflammation along the Alzheimer's disease continuum. J. Neuroinflamm. 16:46. doi: 10.1186/s12974-019-1399-2

Parhizkar, S., Arzberger, T., Brendel, M., Kleinberger, G., Deussing, M., Focke, C., et al. (2019). Loss of TREM2 function increases amyloid seeding but reduces plaque-associated ApoE. Nat. Neurosci. 22, 191-204. doi: 10.1038/s41593-018-0296-9

Pascoal, T. A., Benedet, A. L., Ashton, N. J., Kang, M. S., Therriault, J., Chamoun, M., et al. (2021). Microglial activation and tau propagate jointly across braak stages. Nat. Med. 27, 1592-1599. doi: 10.1038/s41591-021-01456-w

Piccio, L., Buonsanti, C., Cella, M., Tassi, I., Schmidt, R. E., Fenoglio, C., et al. (2008). Identification of soluble TREM-2 in the cerebrospinal fluid and its association with multiple sclerosis and CNS inflammation. Brain 131, 3081-3091. doi: 10.1093/brain/awn217
Piccio, L., Deming, Y., Del-Águila, J. L., Ghezzi, L., Holtzman, D. M., Fagan, A. M., et al. (2016). Cerebrospinal fluid soluble TREM2 is higher in Alzheimer disease and associated with mutation status. Acta Neuropathol. 131, 925-933. doi: 10.1007/s00401-016-1533-5

Rauchmann, B. S., Sadlon, A., Perneczky, R., and Alzheimer's Disease Neuroimaging Initiative (2020). Soluble TREM2 and inflammatory proteins in Alzheimer's disease cerebrospinal fluid. J. Alzheimers. Dis. 73, 1615-1626. doi: 10.3233/JAD-191120

Rauchmann, B. S., Schneider-Axmann, T., Alexopoulos, P., Perneczky, R., and Alzheimer's Disease Neuroimaging Initiative (2019). CSF soluble TREM2 as a measure of immune response along the Alzheimer's disease continuum. Neurobiol. Aging 74, 182-190. doi: 10.1016/j.neurobiolaging.2018.10.022

Schlepckow, K., Kleinberger, G., Fukumori, A., Feederle, R., Lichtenthaler, S. F., Steiner, H., et al. (2017). An Alzheimer-associated TREM2 variant occurs at the ADAM cleavage site and affects shedding and phagocytic function. EMBO Mol. Med. 9, 1356-1365. doi: 10.15252/emmm.201707672

Schlepckow, K., Monroe, K. M., Kleinberger, G., Cantuti-Castelvetri, L., Parhizkar, S., Xia, D., et al. (2020). Enhancing protective microglial activities with a dual function TREM2 antibody to the stalk region. EMBO Mol. Med. 12:e11227. doi: 10.15252/emmm.201911227

Sheng, X., Yao, Y., Huang, R., Xu, Y., Zhu, Y., Chen, L., et al. (2021). Identification of the minimal active soluble TREM2 sequence for modulating microglial phenotypes and amyloid pathology. J. Neuroinflamm. 18:286. doi: 10.1186/s12974-021-02340-7

Song, W. M., Joshita, S., Zhou, Y., Ulland, T. K., Gilfillan, S., and Colonna, M. (2018). Humanized TREM2 mice reveal microglia-intrinsic and extrinsic effects of R47H polymorphism. J. Exp. Med. 215, 745-760. doi: $10.1084 /$ jem.20171529

Suárez-Calvet, M., Araque Caballero, M. Á., Kleinberger, G., Bateman, R. J., Fagan, A. M., Morris, J. C., et al. (2016). Early changes in CSF sTREM2 in dominantly inherited Alzheimer's disease occur after amyloid deposition and neuronal injury. Sci. Transl. Med. 8, 369ra178. doi: 10.1126/scitranslmed.aag1767

Suárez-Calvet, M., Morenas-Rodríguez, E., Kleinberger, G., Schlepckow, K., Araque Caballero, M. Á., Franzmeier, N., et al. (2019). Early increase of CSF sTREM2 in Alzheimer's disease is associated with tau relatedneurodegeneration but not with amyloid- $\beta$ pathology. Mol. Neurodegener. 14:1. doi: 10.1186/s13024-018-0301-5

Thornton, P., Sevalle, J., Deery, M. J., Fraser, G., Zhou, Y., Ståhl, S., et al. (2017). TREM2 shedding by cleavage at the H157-S158 bond is accelerated for the Alzheimer's disease-associated H157Y variant. EMBO Mol. Med. 9, 1366-1378. doi: $10.15252 / \mathrm{emmm} .201707673$

Vilalta, A., Zhou, Y., Sevalle, J., Griffin, J. K., Satoh, K., Allendorf, D. H., et al. (2021). Wild-type sTREM2 blocks $A \beta$ aggregation and neurotoxicity, but the Alzheimer's R47H mutant increases A $\beta$ aggregation. J. Biol. Chem. 296:100631. doi: 10.1016/j.jbc.2021.100631

Wang, S., Mustafa, M., Yuede, C. M., Salazar, S. V., Kong, P., Long, H., et al. (2020). Anti-human TREM2 induces microglia proliferation and reduces pathology in an Alzheimer's disease model. J. Exp. Med. 217:e20200785. doi: $10.1084 / \mathrm{jem} .20200785$

Wang, Y., Ulland, T. K., Ulrich, J. D., Song, W., Tzaferis, J. A., Hole, J. T., et al. (2016). TREM2-mediated early microglial response limits diffusion and toxicity of amyloid plaques. J. Exp. Med. 213, 667-675. doi: 10.1084/jem.20151948

Wu, K., Byers, D. E., Jin, X., Agapov, E., Alexander-Brett, J., Patel, A. C., et al. (2015). TREM-2 promotes macrophage survival and lung disease after respiratory viral infection. J. Exp. Med. 212, 681-697. doi: $10.1084 /$ jem.20141732

Wunderlich, P., Glebov, K., Kemmerling, N., Tien, N. T., Neumann, H., and Walter, J. (2013). Sequential proteolytic processing of the triggering receptor expressed on myeloid cells-2 (TREM2) protein by ectodomain shedding and $\gamma$-secretase-dependent intramembranous cleavage. J. Biol. Chem. 288, 33027-33036. doi: 10.1074/jbc.M113. 517540

Yang, J., Fu, Z., Zhang, X., Xiong, M., Meng, L., and Zhang, Z. (2020). TREM2 ectodomain and its soluble form in Alzheimer's disease. J. Neuroinflamm. 17:204. doi: 10.1186/s12974-020-01878-2

Yuan, P., Condello, C., Keene, C. D., Wang, Y., Bird, T. D., Paul, S. M., et al. (2016). TREM2 haplodeficiency in mice and humans impairs the microglia barrier function leading to decreased amyloid compaction and severe axonal dystrophy. Neuron 90, 724-739. doi: 10.1016/j.neuron.2016.05.003 
Zhao, Y., Wu, X., Li, X., Jiang, L. L., Gui, X., Liu, Y., et al. (2018). TREM2 is a receptor for beta-amyloid that mediates microglial function. Neuron 97, 1023-1031. doi: 10.1016/j.neuron.2018.01.031

Zhong, L., and Chen, X. F. (2019). The emerging roles and therapeutic potential of soluble TREM2 in Alzheimer's disease. Front. Aging Neurosci. 11:328. doi: 10.3389/fnagi.2019.00328

Zhong, L., Chen, X. F., Wang, T., Wang, Z., Liao, C., Wang, Z., et al. (2017). Soluble TREM2 induces inflammatory responses and enhances microglial survival. J. Exp. Med. 214, 597-607. doi: 10.1084/jem.201 60844

Zhong, L., Wang, Z., Wang, D., Wang, Z., Martens, Y. A., Wu, L., et al. (2018). Amyloid-beta modulates microglial responses by binding to the triggering receptor expressed on myeloid cells 2 (TREM2). Mol. Neurodegener. 13:15. doi: 10.1186/s13024-018-0247-7

Zhong, L., Xu, Y., Zhuo, R., Wang, T., Wang, K., Huang, R., et al. (2019). Soluble TREM2 ameliorates pathological phenotypes by modulating microglial functions in an Alzheimer's disease model. Nat. Commun. 10:1365. doi: 10.1038/s41467-019-09118-9
Conflict of Interest: The authors declare that the research was conducted in the absence of any commercial or financial relationships that could be construed as a potential conflict of interest.

Publisher's Note: All claims expressed in this article are solely those of the authors and do not necessarily represent those of their affiliated organizations, or those of the publisher, the editors and the reviewers. Any product that may be evaluated in this article, or claim that may be made by its manufacturer, is not guaranteed or endorsed by the publisher.

Copyright (c) 2022 Brown and St George-Hyslop. This is an open-access article distributed under the terms of the Creative Commons Attribution License (CC BY). The use, distribution or reproduction in other forums is permitted, provided the original author(s) and the copyright owner(s) are credited and that the original publication in this journal is cited, in accordance with accepted academic practice. No use, distribution or reproduction is permitted which does not comply with these terms. 